\title{
Immersive Safety Handling and Training
}

\author{
Håvard Sjøvoll and Egil Tjåland \\ Department of Petroleum Engineering and Applied Geophysics \\ The Norwegian University of Science and Technology \\ S.P. Andersens vei 15A, N-7491 Trondheim, Norway \\ e-mail: \{sjovoll/egil.tjaland\}@ntnu.no
}

(Rec. 15 May 2006)

\begin{abstract}
A central challenge for the oil industry is to make correct decisions in abnormal, complex and time-critical settings. This is to ensure safety, regularity and optimized operation and production. A new information platform has been developed to identify and localize unwanted events, and to train control room operators for optimal decision making. This platform has been implemented in an immersive CAVE environment to ensure a realistic setting and a better understanding of the problem. The system reports unwanted events in a 3D environment, and the user may obtain error handling procedures to rectify the given problem. These procedures are retrieved by semantic searches in an ontology. The ontology is stored in a central repository, and accessed through a client-server connection.
\end{abstract}

Key words: Integrated Operations, immersive, Virtual Reality, safety, training, Semantic Web

\section{INTRODUCTION}

$E$-field and Integrated Operations ${ }^{1}$ is the next generation of efficiency development for the entire oil and gas industry. The result of the full implementation of IO is a business transformation that will influence the oil and gas companies at all levels: advanced technology applications, organizational structures and work processes, and the interaction between all parties involved both inside and outside the oil companies. The oil companies and the service companies will work in a much closer relationship in the future. One important change is the new Onshore Support Centers (OSC) that supplements, and in some cases take over, the traditional control structures at the offshore oil and gas installations. This transformation of control from the offshore rigs to onshore control centers requires a strong focus on safety handling and training. The use of Virtual Reality centers to train control room personnel to handle simulated offshore operations is an appealing way to maintain the strict safety regulations demanded in the oil and gas industry.

Existing studies on training of control room operators have concentrated on team development and training on such diverse teams as aircraft cockpit crews, military combat center groups and nuclear power plants operators [1], in which the former resembles the subjects in our case.

The international aviation industry has been using a special form of human factors training with their flight deck crews and other teams, called crew resource manage-

\footnotetext{
${ }^{1} e$-field and Integrated Operations is abbreviated by IO
}

ment (CRM). CRM is designed by psychologists and pilots to reduce errors and accidents and to improve emergency response capability by improving teamwork skills. This approach has successfully been used for training of control room operators and emergency command teams in the offshore oil industry, with promising results regarding enhanced safety and improved productivity [2].

Some studies have advocated the use of special algorithms to optimize decision taking in complicated settings. A so-called fisheye view, generated by a "variable-zoom" algorithm has been shown to simplify the problem for navigating in a simulated network [3]. It was shown that users were quicker to complete their tasks and that they made fewer unnecessary navigational steps through a network hierarchy using the fisheye view compared to a fullzoom technique.

Studies have shown that multimodal user interaction may be useful for control room operators where no obvious satisfactory solution can be found using classical graphical user interface techniques [4]. An immersed environment could easily utilize this finding.

When it comes to semantic web technology, much research has been done in this area which is relevant for this paper. However, very few of them advocate the combination of semantic web and Virtual Reality. Kalogerakis [5] shows an interoperable framework for the integration of virtual reality scenes with semantic information. This includes methodologies and tools for the exploitation of this framework, like semantic querying, interaction, personalization and construction of scenes with inference. In [6], 
Cera et al. presents an approach to collaborative authoring of design semantics within a multi-user 3D environment. The Multi-user Groups for Conceptual Understanding and Prototyping (MUG)-platform enables designers to collaborate over the Internet in a shared 3D workspace. This is done in order to support knowledge-based conceptual design, using a Semantic Web representation framework.

In this paper we demonstrate how an immersed environment can be used to train control room operators and provide better support for making optimal decisions. The developed system which is presented is a proof-of-concept for a near real time decision support system, utilizing an immersive environment and semantic technology.

\section{INTEGRATED INFORMATION PLATFORM}

The Integrated Information Platform (IIP) project is an $\mathrm{R} \& \mathrm{D}$ project that is supported by the Norwegian Research Council, and aims to resolve one of the establishments of a real-time information pipeline that supports the future generations of IO [7]. An optimal set of real time data from reservoirs, wells and subsea production facilities will be identified, partially improved and integrated to provide an open and standardized information platform. This platform makes it possible for multi-disciplinary teams to work simultaneously in an integrated operation environment that may consist of many operation centers geographically dispersed over wide distances.

The project aims to achieve this through development of underlying technology and standards:

- Machine-interpretable terminologies for relevant domains forming the basis for development of XML schemas for consistent transferal of data between applications in same domain.

- Taxonomies for multiple domains or combination of data across domains.

- Complete ontologies supporting automated reasoning or inference of data using logical rules.

- A Reference Data Library (RDL) containing terminologies, taxonomies and ontologies.

The project addresses the following domains: Reservoir characterization, drilling and logging, well production, safety and automation systems, subsea production equipment, condition based monitoring and health, safety and environment.

The semantics developed - terminologies, taxonomies and ontologies - is based on ISO 15926: "Integration of life-cycle data for process plants including oil and gas production facilities". ISO 15926 defines a language that both applications and professionals can understand. The philosophy that this concept is based on is compatible with W3C's Semantic Web.

The research within IIP is a contribution towards better IO. In [8], Fjellheim and Norheim claims that the management of offshore oil and gas fields, in the North Sea and elsewhere, is moving towards IO, i.e. integrated work processes, real-time data from the field, on-shore operation centers, and massive use of IT for on-line monitoring, analysis, and decision tasks. It was also the conclusion of the Better Resource Utilization (BRU)-report [9], a strategic plan for focused petroleum research at NTNU, that currently $\mathrm{IO}$ is one of the most important issues in the petroleum industry, and that it will be the next efficiency step and breakthrough for the entire global petroleum industry.

\subsection{Semantic Web and Ontologies}

The Semantic Web, with its vision stated by Berners-lee et al. [10], aims at developing languages for expressing information in a machine understandable form. Until recently, the World Wide Web has been more about documents for humans rather than information that can be automatically manipulated. By adding metadata to these documents, the Web can become the Semantic Web. The recent explosion of interest in the World Wide Web has also fuelled interest in ontologies [11]. It has been predicted that ontologies will play an important role in the Semantic Web since ontologies enable shared domain models, which are understandable to both machines and humans [12].

In classic philosophy, an ontology is the most fundamental branch of metaphysics. It tries to find the relation between entities as well as studies of being and existence, and the concept of reality is strongly implicated. Typically, the result is laid out in a hierarchical way. However, it does not need to be hierarchical. It may be described in a cyclical way, much like the nature of the universe itself.

An ontology in computer science terms is derived from ontology in philosophy. It is the description of a domain which is done in a thorough and strict fashion. The result is a data structure containing the entities and their relationship within the domain at hand.

There are basically two types of ontologies:

- Domain ontologies are strongly tied to a specific domain, and are made with the necessary level of detail.

- Foundation ontologies are less specific than domain ontologies and are thus more general applicable.

The functionality of a computational system that utilizes an ontology is dependent not only on the structure and the data within the ontology, but also on the software implementation.

The anatomy of an ontology is explained as follows:

- Concepts are anything you can say about something, be it real or fictitious. It may be concrete or abstract, a task, a reasoning process, a function etc. A simple example to illustrate this is depicted in Fig. 1.

- Attributes can be assigned to every concept in the ontology. Attributes allow more complex relationships modeled using the ontology. 
Our approach conducts the domain ontology path, using a specific ontology which describes physical components

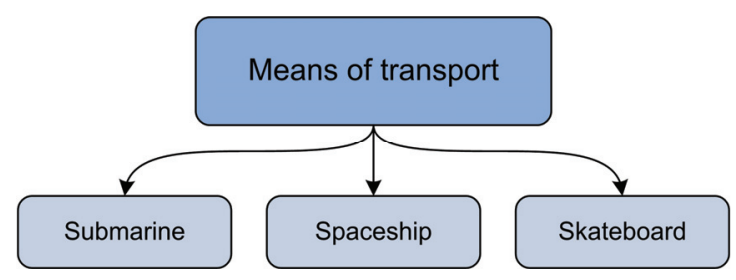

Fig. 1. Example of concepts in an ontology

on oil rigs, and their connections. The document base containing all the information will potentially be large. The operations may be time critical. Therefore it is of importance to show relevant subsets to the operator.

\section{DESIGN AND IMPLEMENTATION}

This section describes the overall design and implementation of the Integrated Information Platform.

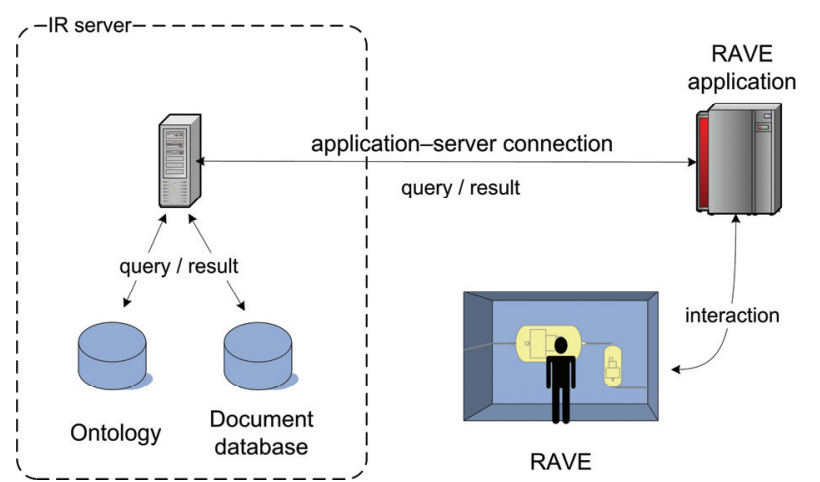

Fig. 2. Overall design of the Integrated Information Platform

As depicted in Fig. 2, the platform consists of several components, each playing a major role for the system to operate; 1) the Information Retrieval server with the document base and the ontology, 2) the RAVE application, 3) the application-server connection, and finally 4) the RAVE in which the program is presented to the user. Each of these components will now be briefly introduced.

\subsection{Information Retrieval Server}

The Information Retrieval (IR) server communicates with the RAVE application in classic client-server architecture, and returns answers to the application's requests. The server is written in Java and consists of several classes used to handle execution, communication and searching. The server handles multiple requests simultaneously by means of threads. A class diagram of the IR server is shown in Fig. 3.

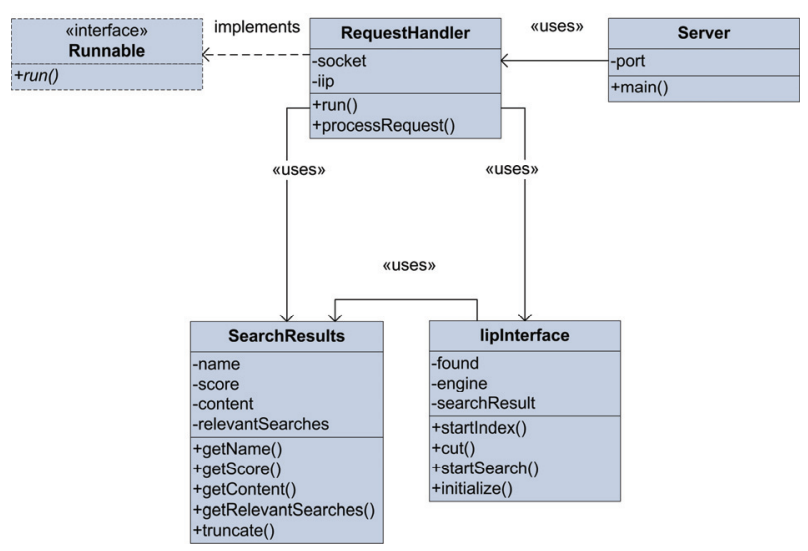

Fig. 3. Class diagram of IR server

The most important parts of the server are handled by the IR framework, which includes ontology and document searching and indexing. Based on one or more searchkeywords, the IR framework returns hits in documents and a list of relevant objects/keywords. These keywords could, in addition to describe physical objects, also describe the state of a facility or measured values. For instance the search-result could differ if the state of the facility were at a critical stage.

The documents consist of a parsed and indexed collection of text-files, like routine-descriptions, technical specifications and logs. Only the PDF and HTML file formats are supported, but new parsers may be written.

The ontology is defined in OWL and corresponds to the 3D-model(s) loaded in the RAVE-application, such that object-names are used consistently.

\subsection{RAVE Application}

This program is written in $\mathrm{C}++$, using OpenGL Performer and CAVELib. Performer [13] is a scene graphbased library developed by SGI, designed to simplify the development of applications used for virtual reality. Performer also supports multiple processor systems and multiple graphic pipelines, which are useful when working in a RAVE environment. Performer has been used to build the scene graph by importing the different models in the system, and to handle the naming of objects, relations, etc. VRCO CAVELib is, according to VRCO, the most widely used commercial API for developing immerse, interactive 3D (i3D) visualization applications [14]. Using CAVELib also enables the application to be executed in a non-RAVE environment, such as a desktop PC, by simulating the tracker-/wand-input and the immersive visualization.

The program creates the virtual environment, consisting of the facility to be operated, and provides a 3D user interface to the operator, as depicted in Fig. 4. 


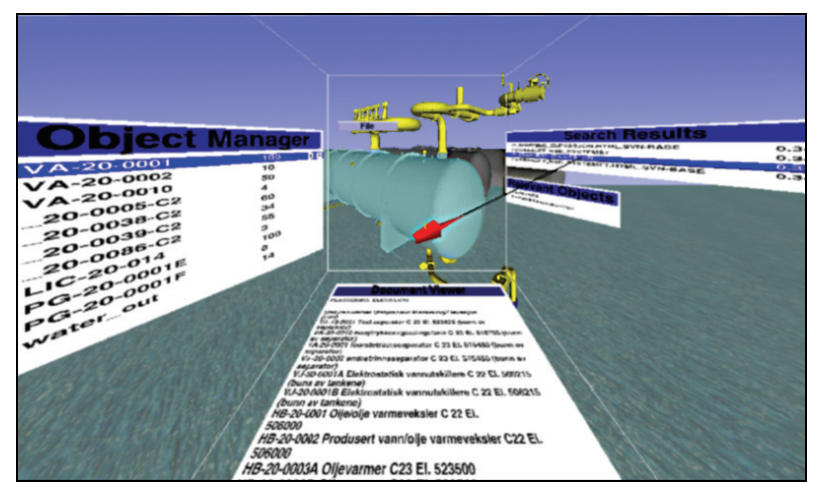

Fig. 4. RAVE application in simulator-mode, showing all projected walls within the RAVE

The operator selects objects and interacts with the program with a wand. When the operator points the wand towards an object, the object will be highlighted by a change of color. An object list, projected on the left wall, also indicates which object is selected, and provides tools for altering the properties of the object (like opening a valve). Whenever an object is selected, the IR-server performs a semantic search based on the object and the state of the system, and returns a sorted list of relevant objects and documents. The list is shown on the right wall. If the list is clicked, the program will retrieve the actual document and projects it on the floor.

\subsection{Connection}

Whenever a request is made by the operator, a search string is transferred to the IR-server by means of a TCP/IP connection. The HyperText Transfer Protocol (HTTP) is used in the application-layer of the communication, ensuring compatibility and modularity towards other systems. The returning results from the server are formatted and transferred in the eXtensible Markup Language (XML).

On the client-side (RAVE-application), two classes, based on open source projects, are used to send and receive data; Net and Parser. The Net-class uses PracticalSocket [15] to create a TCP-socket. Parser utilizes TinyXML [16] to parse the XML received from the IR-server.

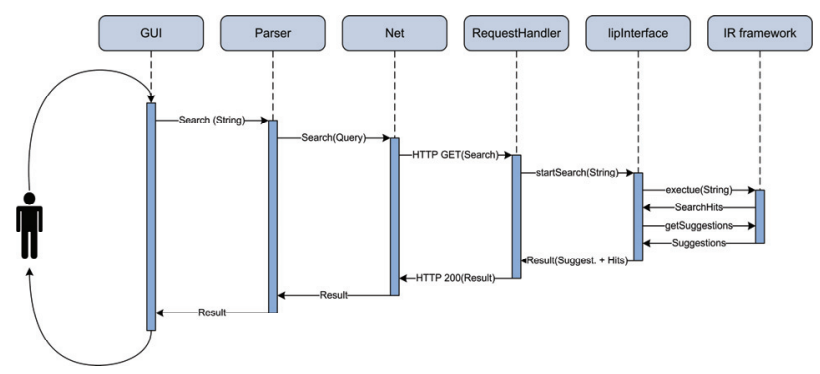

Fig. 5. Search sequence diagram of the Integrated Information Platform
Whenever a request is made from the client, the server creates a new socket and a thread to handle the request. The thread reads the request, finds the search query and performs a search. The result is converted to a XML-string and returned to the client. A search-sequence diagram is depicted in Fig. 5.

\subsection{RAVE}

In May 2001, the Department of Petroleum Engineering and Applied Geophysics at NTNU installed the first large scale visualization laboratory, and the first CAVE-solution, at a Norwegian university.

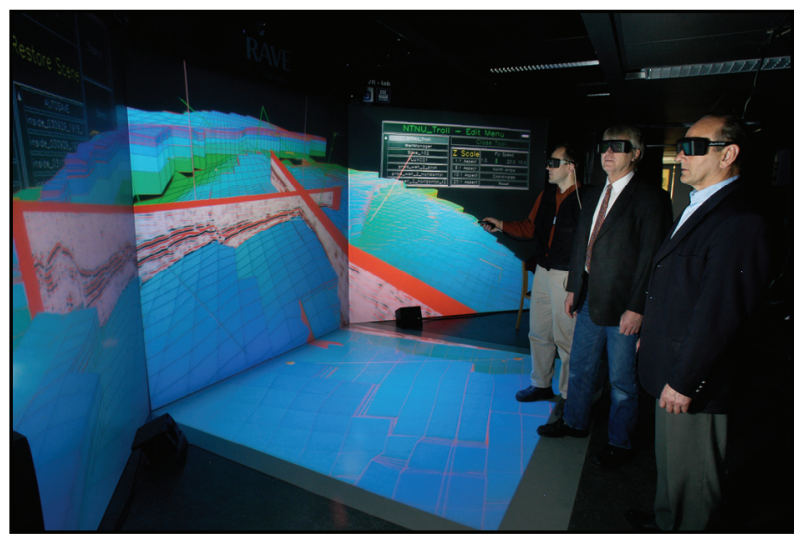

Fig. 6. The RAVE at the Department of Petroleum Engineering and Applied Geophysics at NTNU. Courtesy of E. Tjåland [17]

The key element of the Collaboration Learning Virtual Reality (CLVR)-lab is the RAVE, a reconfigurable unit manufactured by Fakespace Inc (see Fig. 6). The unit consists of 3 separate modules, one of which with floor projection. The RAVE may be configured as a 4-walled $2.5 \mathrm{~m}$ cubed CAVE, a $7.5 \mathrm{~m}$ by $2.5 \mathrm{~m}$ flat wall, a theatre with arbitrary angle, or may be used as 3 independent flat walls of $2.5 \mathrm{~m}$ squared. Thus, the unit may be used for total immersion for a small group of people, as a theatre, as a large flat screen for a large group of people, or as 3 independent flat screens used by project groups.

The computer driving the RAVE is a 16-CPU SGI Onyx 4 with 8 ATI FireGL X2 pipes, each with $256 \mathrm{MB}$ RAM. In addition to this, two Linux-workstations equipped with the nVidia QuadroFX 3000G graphics card, are also connected and can utilize the RAVE.

The RAVE is, beside research projects, mainly used for education and visualization of data from reservoir simulation, seismics and medicin.

\section{SAFETY HANDLING AND TRAINING}

A high amount of complex and urgent information needs timely attention in an operational environment. This re- 
quires specialized systems. These systems should provide immediate access to accurate and pertinent information when troubleshooting or controlling abnormal situations. The Integrated Information Platform described in this paper suggests using an immersive environment to train control room operators for optimal decision making. Within immersive technology like Virtual Reality, training has been a very popular area of research due to its advantages on behalf of cost and safety compared to real environments. Louka and Balducelli claims in [18] that VR-based training is particularly well suited to situations where cognitive and spatial skills are important. Flight- or crane simulators are examples of such.

For a control room operator, the spatial learning provided by IIP cannot directly be used since today's systems do not utilize this. In other words, it is not common for a control room operator to rectify abnormal situations at the physical location of the problem. Control rooms exist to improve the efficiency of such operations. However, if physical intervention is necessary, spatial knowledge of the facility will be of great importance for the operator who directs the field-operators. It can also be argued that better understanding and knowledge of the physical properties of the facility, like spatial information, dimensions and distances, will improve the operator's ability to troubleshoot and solve problems. Taking into account that many facilities also consists of subsea equipment only fortifies this point.

One of the true advantages of IIP is the way the operator can request and retrieve relevant information. The complexity of the systems under control can be vast, and immediate access to documentation, knowledgebase, routines and specifications is important. By use of semantic technology the retrieval of such information can be made more effective than today's systems. Even though it is not within the scope of this paper to go into detail of this technology, further development within this area will be of great importance for the industry. The system is also scalable and the architecture allows for multiple operators and/or control rooms to use the same IR-server. This client-server architecture also enables the possibility of collaborative work amongst operators. Within IO this is important to allow different experts to contribute in problem-solving and thus makes new work processes and organizational models possible and/or essential.

\section{CONCLUSION}

In an oil and gas industry where operations are rapidly moving from offshore to onshore control units, it is important to have control room operators with adequate skills to handle challenges in an efficient and safe way. To achieve this objective we have here shown a strategy to train con- trol room operators in an immersive virtual setting using an underlying integrated information platform that can be accessed as needed. The information platform is based on ISO 15926 which applies to seismic, drilling, equipment, production, operation, maintenance and safety information for subsea systems. The training simulator is designed to incorporate routine operator work processes as well as crisis management.

The immersed environment gives the user a realistic 3D perspective, well suited to enhance learning and understanding. The defining ontology and access to databases through semantic searches makes the system ideal for making correct and immediate decisions in abnormal and/or critical situations.

\section{Acknowledgments}

The paper is based on work accomplished by students and staff at the Department of Petroleum Engineering and Applied Geophysics, NTNU and the Department of Computer Science, NTNU, in co-operation with Det Norske Veritas (DNV) and the Norwegian oil and gas industry.

This work was supported by the Norwegian Research Council (NFR) through program 163457/S30.

\section{References}

[1] M. T. Brannick, E. Salas and C. Prince (ed.), Team performance assessment and measurement: Theory, methods, and applications. Mahwah, JN: Lawrence Erlbaum 1997.

[2] R. H. Flint, Crew resource management for teams in the offshore oil industry, Journal of European Industrial Training, Vol 19, Issue 9, 23-27 (1995).

[3] D. Schaffer, Z. Zuo, S. Greenberg, L. Bartram, J. Dill, S. Dubs and M. Roseman, Navigating hierarchically clustered networks through fisheye and full-zoom methods. ACM Transactions on Computer-Human Interaction 3(2), 162-188 (1996).

[4] F. Chen, E. H. C Choi, N. Ruiz, Y. Shi and R. Taib, User Interface Design and Evaluation for Control Room, Proceedings of OZCHI 2005, Canberre, Australia, Nov. 23-25 (2005).

[5] E. Kalogerakis, S. Christodoulakis and N. Moumoutzis, Coupling Ontologies with Graphics Content for Knowledge Driven Visualization in: B. Frölich, D. Bowman and H. Iwata (ed.) Proceedings of IEEE Virtual Reality 2006, pp. 43-50 (2006).

[6] C. . Cera, W. C. Regli, I. Braude, Y. Shapirstein and C.V. Foster, In: A Collaborative 3D Environment for Authoring Design Semantics, IEEE Computer Graphics and Applications, Special Issue on "Computer-Aided Design", 22(3), 42-55 (2002).

[7] N. Sandsmark and S. Mehta, Integrated Information Platform for Reservoir and Subsea Production Systems, POSC Caesar Assosiation (2004).

[8] R. Fjellheim and D. Norheim, AKSIO - An application of Semantic Web technology for knowledge management in the petroleum industry, ISWC2005 Poster Submission (2005). 
[9] J. Kleppe, M. Landrø, B. Ursin, S. Sangesland and A. Nystad, BRU-report, Internal publication at The Norwegian University of Science and Technology (2005).

[10] T. Berners-Lee, J. Hendler and O. Lassila, The Semantic Web, Scientific American, May (2001).

[11] J. Z. Pan and I. Horrocks. Metamodeling architecture of web ontology languages, Proceedings of the Semantic Web Working Symposium, Stanford, 131-149, July (2001).

[12] J. Broekstra, M. Klein, S. Decker, D. Fensel, F. van Harmelen and I. Horrocks, Enabling knowledge representation on the Web by Extending RDF Schema, Proceedings of the Tenth International World Wide Web Conference (2001).

[13] SGI. OpenGL Performer [online]. Available from: http:// www.sgi.com/products/software/performer/
[14] VRCO. CAVELib [online]. Available from: http://www.vrco.com/CAVELib/OverviewCAVELib.html

[15] Jeff Donahoo, Practical C++ sockets [online]. Available from: http://cs.ecs.baylor.edu/ donahoo/ practical/CSockets/practical/

[16] TinyXml Projet. Tinyxml package [online]. Available from: $\mathrm{http} / / /$ www.grinninglizard.com/tinyxml/

[17] E. Tjåland, Visualization of Real-time Production Data from Subsea Installations, SGIUG 2005, Munich, Session C-7 VIZ (2005).

[18] M. N. Louka and C. Balducelli, Virtual Reality Tools for Emergency Operation Support and Training In Proceedings of TIEMS 2001 (The International Emergency Management Society), Oslo, June (2001).

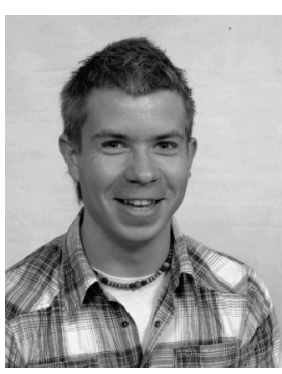

HÅVARD SJØVOLL was born in 1979 and received his M.Sc. degree in computer science at the Department of Computer Science, NTNU in Trondheim, Norway in 2005. He started his Ph.D. studies at Department of Petroleum Engineering and Applied Geophysics, NTNU in 2005. His research interests include multi-modal human-computer interaction, immersive environments and integrated operations. He is a member of IEEE (2005), ACM/SIGGRAPH (2005) and EAGE (2006).

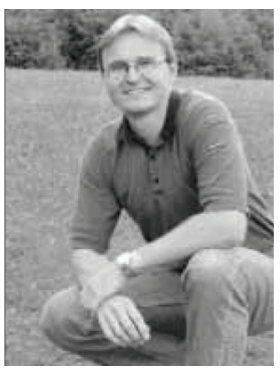

EGIL TJÅLAND was born in 1961 and received his M.Sc. degree in geophysics at The Norwegian University of Science and Technology (NTNU) in 1986 and a Ph.D. in 1994, also in geophysics. His research interests are seismic source development, integrated operations and visualization. He is Senior Member of EAGE and SEG and a Member of AAPG. 